\title{
Gradhiva
}

GRADHIV

Revue d'anthropologie et d'histoire des arts

$23 \mid 2016$

Collections mixtes

\section{Genèses de La Fin du monde de De Martino}

The origins of De Martino's The End of the World

\section{Carlo Ginzburg}

Traducteur : Camille Joseph

\section{(2) OpenEdition}

Journals

Édition électronique

URL : http://journals.openedition.org/gradhiva/3187

DOI : 10.4000/gradhiva.3187

ISSN : 1760-849X

Éditeur

Musée du quai Branly Jacques Chirac

Édition imprimée

Date de publication : 25 mai 2016

Pagination : 194-213

ISBN : 978-2-35744-093-7

ISSN : 0764-8928

\section{Référence électronique}

Carlo Ginzburg, "Genèses de La Fin du monde de De Martino », Gradhiva [En ligne], 23 | 2016, mis en ligne le 25 mai 2019, consulté le 03 mai 2019. URL : http://journals.openedition.org/gradhiva/3187 ; DOl : 10.4000/gradhiva.3187 


\section{Genèses de La Fin du monde de De Martino'1}

par Carlo Ginzburg

Cet article analyse le dernier projet de recherche,

1. Ce texte est issu d'une conférence donnée le inachevé, d'Ernesto De Martino: ses notes parues sous le titre La Fin du monde dans une édition posthume. Nous remontons ici aux sources lointaines du projet, au moment où est conçu Le Monde magique, dans les années 1940, alors que De Martino découvre les travaux de Sergueï Shirokogoroff sur les chamanes toungouses et - à travers Wilhelm Emil Mühlmann, chercheur nazi en folklore et en anthropologie - la pensée de Martin Heidegger. C'est dans ce contexte que De Martino élabore sa thèse fondamentale sur la perte de la présence. Mais c'est seulement au début des années 1960 que celle-ci est reliée au thème de la fin du monde, grâce à un film, ou plutôt aux dernières minutes d'un film: L'Éclipse (1962) de Michelangelo Antonioni. 
2. Ernesto De Martino, Le Monde magique, Paris, Les Empêcheurs de penser en rond, 2003 [// mondo magico. Prolegomeni a una storia del magismo, Turin, Einaudi, 1948]. Le manuscrit fut envoyé à l'éditeur, Giulio Einaudi, le 8 août 1946: voir id. Dal laboratorio del «Mondo magico». Carteggi 1940-1943, Pietro Angelini (éd.). Lecce, Argo, 2007, p. 9. Le livre a été traduit en plusieurs langues: anglais, français, espagnol, tchèque, hongrois, polonais et japonais.

3. Les passages cités sont traduits en français à partir de fragments non publiés d'Ernesto De Martino. On trouve une autre allusion rétrospective, mais plus évidente cette fois, à sa trajectoire personnelle dans la remarque suivante: "Le primitif, le barbare, le sauvage n'étaient pas autour de moi car, parfois, il m'arrivait avec angoisse d'entendre aussi au-dedans de moi résonner des voix archaïques, et fermenter des inclinaisons et des incitations à adopter des comportements gratuits, irrationnels, inquiétants: quelque chose de chaotique et de trouble, qui réclamait l'ordre et la lumière. C'est ainsi que mûrit une série de pensées et de recherches qui aboutirent à la thèse fondamentale du Monde magique, etc. " (Ernesto De Martino, «Etnologia e cultura nazionale» [«Ethnologie et culture nationale "], Società 9, 1953: 314-315)

\section{ci-contre}

fig. 1

Jaquette de la couverture de La fine del mondo: contributo all'analisi delle apocalissi culturali. Turin, Einaudi, 1977.
On peut considérer La Fin du monde, dernier projet inachevé d'Ernesto De Martino, comme le fruit d'incitations diverses, en partie contradictoires. J'essaierai d'en reconstruire quelques-unes, en m'appuyant tant sur des évidences que sur des conjectures raisonnablement contextualisées.

\section{I}

1. Le trait le plus évident du projet de La Fin du monde est sa relation avec Le Monde magique, le livre le plus original et le plus dérangeant de De Martino ${ }^{2}$. La magie, affirme l'auteur, contribue puissamment à rétablir la présence, fragile et menacée, de l'individu dans le monde. En un sens, suggère De Martino, le monde en tant que tel, et la présence de l'homme au monde, sont des produits historiques de la magie. Il y a longtemps, développant une idée avancée par Renato Solmi, j'ai relevé certaines analogies entre Le Monde magique de De Martino et La Dialectique de la raison de Theodor W. Adorno et Max Horkheimer qui m'ont conduit à inscrire ces deux ouvrages dans une catégorie plus large, les «livres de l'année zéro" (Ginzburg 1979: 238-242; d'autres commentaires: 228-248). J'adopterai ici une perspective différente, mais nullement incompatible. Un fragment publié par Giordana Charuty dans sa biographie fondamentale, Les Vies antérieures d'un anthropologue, montre que De Martino était pleinement conscient de l'ancrage personnel et émotionnel des arguments qu'il développait dans Le Monde magique. Jeune, il souffrait en effet de crises d'épilepsie qu'il s'employa rétrospectivement à interpréter dans le cadre de sa propre notion de "perte de la présence»:

Lorsque j'analyse l'expérience de mon «aura», il me semble remarquer avant tout une profonde dysthymie. L'aura commence ainsi: le monde devient sordidement étranger, diaboliquement menaçant, il se dépouille de sentiments. C'est le signe que la présence commence à s'affaiblir. [...] Puis, après quelques instants, la présence émerge du naufrage et, avec elle, le monde restauré dans ses formes, avec ses sentiments. C'est comme si je glissais lentement hors de l'histoire ${ }^{3}$. (Charuty 2009: 57-59)

Afin d'éviter tout malentendu, précisons tout de suite que je m'abstiendrai de lire l'œuvre de De Martino comme le produit de ses maladies: ce serait une conception naïve et fausse du biographique. Cependant, l'histoire et l'anthropologie sont des formes situées de savoir. À travers son expérience personnelle, intellectuelle et émotionnelle (qui inclut ses maladies), De Martino a élaboré des propositions qui se veulent de portée générale et qu'il nous faut évaluer à partir de leur domaine propre. Sa réflexion sur le naufrage du monde qui menace l'individu a donné lieu, vingt ans plus tard, à une interrogation sur la fin du monde qui menace l'espèce humaine tout entière. Ce faisant, il revenait à son premier livre pour en développer quelques implications dans un contexte entièrement différent. Dans les deux cas, les phénomènes pathologiques lui ont servi de clé pour rendre compte de configurations plus vastes, dépourvues de toute dimension pathologique. Ce saut interprétatif mérite une analyse détaillée.

2. Au tout début du Monde magique, le lecteur rencontre la citation de deux pages d'un livre qui demeure un classique de la littérature 
ERNESTO DE MARTINO

\section{LA FINE DEL MONDO}

CONTRIBUTO ALL'ANALISI

DELLE APOCALISSI CULTURALI

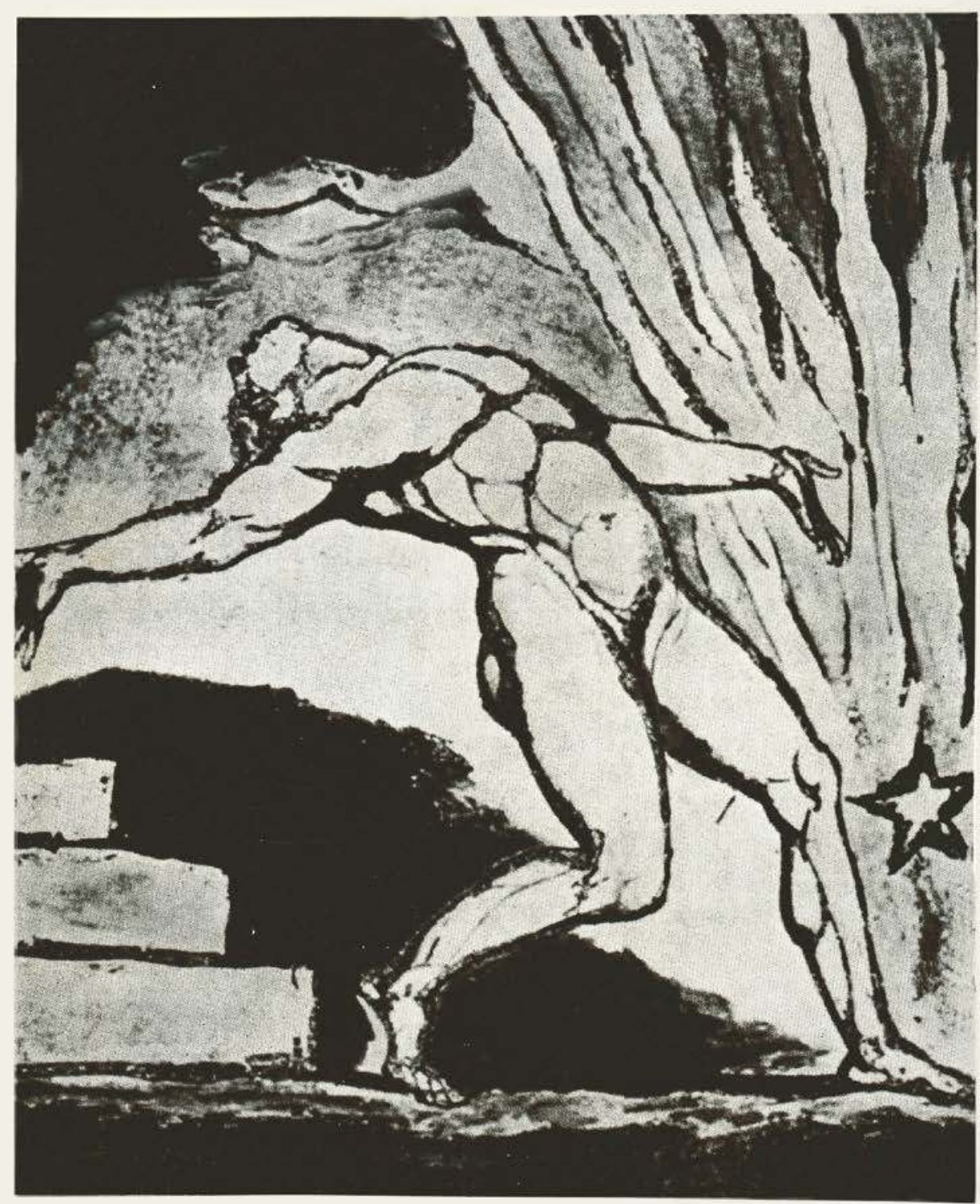




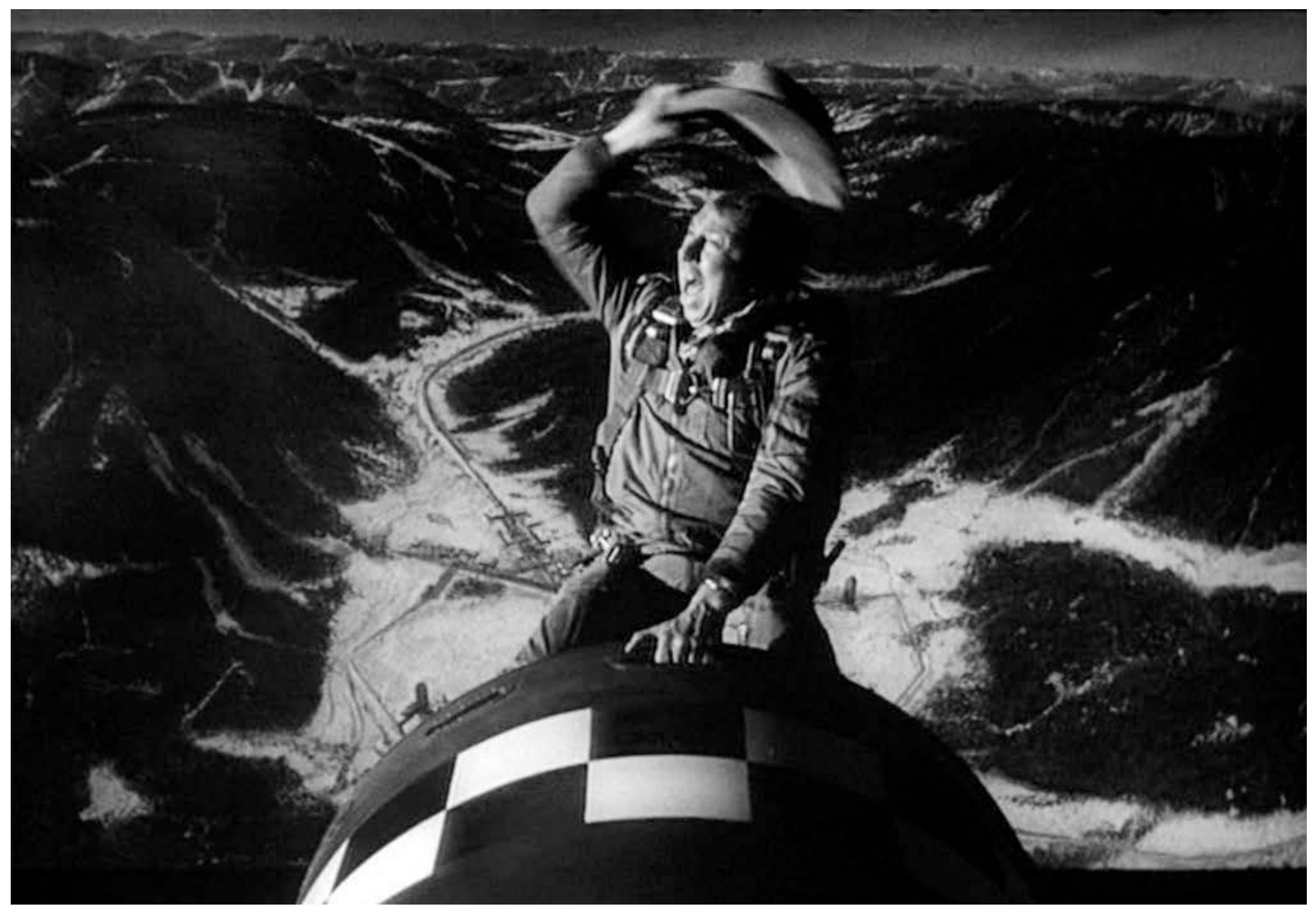

fig. 2

Stanley Kubrick, Docteur

Folamour, 1964 avec Slim

Pickens $\odot$ Photo Rue des

Archives/DILTZ. 
sur le chamanisme, The Psychomental Complex of the Tungus, de Sergueï Shirokogoroff, publié à Londres en 1935. De Martino emprunta l'ouvrage à l'historien des religions Raffaele Pettazzoni et en publia un compte rendu en 1942. Mais il en avait déjà transcrit de longs passages en notes en $1940^{4}$. C'est un autre livre, également cité dans Le Monde magique, qui a, me semblet-il, attiré son attention sur l'ouvrage de Shirokogoroff, à savoir Methodik der Völkerkunde de Wilhelm Emil Mühlmann 5 (Stuttgart, 1938). Naturalisme et historicisme en ethnologie, le premier livre de De Martino, faisait déjà référence à un essai de Mühlmann qu'il présentait avec emphase comme «l'une des contributions théoriques les plus abouties que nous ayons lues en dépouillant la documentation ${ }^{6}{ }$.

Une précision s'impose ici. Mühlmann, auteur d'une histoire de l'anthropologie qui a fait autorité, fit une longue et brillante carrière académique dans le domaine de l'anthropologie et du folklore; les deux bibliographies qui lui ont été consacrées, en 1968 et 1984, ont dûment mentionné ses articles et ouvrages consacrés à la raciologie (Rassenkunde), publiés dans les années 1930 et 1940. On a étudié Mühlmann, un nazi pur et dur, comme un des cas les plus flagrants d'«amnésie et d'amnistie "académiques 7 . Methodik der Völkerkunde, un livre remarquable à bien des égards, cite à plusieurs reprises The Psychomental Complex, chaleureusement présenté comme une étude accomplie d'anthropologie fonctionnaliste fondée sur une approche historique et psychologique. De plus, Mühlmann propose des comparaisons audacieuses avec un autre livre publié à la même époque: Naven de Gregory Bateson ${ }^{8}$. Mühlmann et Shirokogoroff entretinrent des relations personnelles. À la fin des années 1930, Shirokogoroff, qui s'était auto-exilé en Chine en tant qu'opposant au régime soviétique, adressa à Mühlmann des lettres pleines de tirades anti-britanniques, de remarques prudentes sur le développement de la situation politique en Allemagne et de réflexions fort perspicaces sur son propre livre, The Psychomental Complex of the Tungus. En 1940, Mühlmann intégra de longs passages de cette correspondance dans une nécrologie de Shirokogoroff, mort l'année précédente (Mühlmann 1940).

Je reviendrai plus loin sur Methodik der Völkerkunde de Mühlmann et sur la lecture qu'en fit De Martino. Ce qui suscita l'intérêt de ce dernier pour Shirokogoroff, nous le voyons surgir dans un long article dont la première partie, rédigée en 1940, était parue en 1942 (avant de devenir, sous une forme révisée, le premier chapitre du Monde magique). Le chercheur italien était loin de partager l'habituel scepticisme de la grande majorité des ethnologues sur la réalité des pouvoirs magiques au sein des populations dites «primitives». II fallait, pour prendre au sérieux une question aussi scandaleuse, se fonder sur les études de parapsychologie et d'autres phénomènes connexes, domaines qui avaient vivement intéressé le jeune De Martino (Charuty 2009: 254-261; Satta 2005; archives Ernesto De Martino, Rome, 3.8.). Dans cette perspective, De Martino notait de longs passages de The Psychomental Complex qui valaient comme preuve de la réalité des pouvoirs magiques chez les Toungouses.

Shirokogoroff était un relativiste radical et, à ce titre, rejetait l'opposition entre peuples primitifs (Naturvölker) et peuples civilisés, qu'il considérait
4. Rome, archives

De Martino 3.8.26

Charuty 2009: 259, note

65 ; Ernesto De

Martino, "Compte rendu»,

in Studi e materiali di storia delle religioni 17,1942 :

108-111. Voir également

id., "Percezione

extrasensoriale e magismo etnologico " ["Perception extrasensorielle et

magisme ethnologique "],

Studi e materiali di storia

delle religioni 18 ,

1942: 1-19. La publication de cet essai reprend après la guerre: ibid., 19-20, 1943-1946: 31-84. Pour la date de la rédaction, voir p. 5: «Les principaux résultats obtenus jusqu'à présent (1940)». L'auteur connaît déjà le livre de Sergueï Shirokogoroff lorsque le père Michele Schulien lui en suggère la lecture (ce qui a échappé à Pietro Angelini, Ernesto De Martino, Rome, Carocci, 2008: 33-34).

5. Voir De Martino 2003: 93, note 2 (avec une référence erronée à Methodik der Völkerkunde) et 101, note 2 ; d'autres références à Wilhelm Emil MühImann (Rassen- und Völkerkunde. Lebensprobleme der Rassen, Gesellschaften und Völker, Braunschweig Friedr. Vieweg \& Sohn, 1936) in ibid. : 95, note 1 et 169 , note 1 .

\section{Voir Naturalismo e} storicismo nell'etnologia, Bari, Laterza, 1941: 197-198 sur la contribution de MühIman à Konrad Theodor Preuss (éd.), Lehrbuch der Völkerkunde, Stuttgart, Ferdinand Enke Verlag, 1937. Rassenund Völkerkunde... est déjà cité p. 192, note 38 ; voir aussi p. 193-195.

7. Voir Horst Reimann et Klaus Kiefer (éd.), Bibliographie 1928-1964. Wilhelm Emil MühImann zum 60. Geburtstag, Wiesbaden, Harrassowitz, 1964 ; Wilhelm Emil Mühlmann, Geschichte der Anthropologie, Francfort et Bonn, Athenäum Verlag, 1968 :
Horst Reimann (éd.),

Bibliographie 1965-1984. Wilhelm Emil MühImann zum 80. Geburtstag, Augsbourg, Univ.,

Lehrstuhl für Soziologie u. Kommunikationswiss, 1984. Sur l'amnésie, voir Ute Michel, « Wilhelm Emil Mühlmann (19041988) - ein deutscher Professor. Amnesie und Amnestie: Zum Verhältnis von Ethnologie und Politik im Nationalsozialismus ",

Jahrbuch für

Soziologiegeschichte, 1991 ; Carsten Klingemann, Soziologie und Politik.

Sozialwissenschaftliches

Expertenwissen im

Dritten Reich und in der

frühen Westdeutschen Nachkriegszeit, Wiesbaden, Springer, 2009: 363-373. Voir aussi Wilhelm Emil Mühlmann, Rassen- und Völkerkunde..., op. cit. : 536-537 (cité dans Le Monde magique: 95 , note 1 et 169 , note 1 ); après une rapide allusion aux lois de Nuremberg (1935), Mühlmann écrivait: "Une solution [de la question juive] est extrêmement difficile; elle engendre des préoccupations sérieuses. On ne pourra dire quelque chose de plus là-dessus que dans quelques années (Eine Lösung [des Judenproblems] ist unendlich schwer und gibt zu ernsten Sorgen Anlaß. Erst in einigen Jahren wird sich darüber mehr sagen lassen). "S'agissait-il d'une prévision ou d'un souhait?

8. Wilhelm Emil Mühlmann, Methodik der Völkerkunde, Stuttgart, Ferdinand Enke, 1938: 158 et 162-163 sqq. La préface est datée: «Hambourg, 13 mars 1938. Am Tage der Rückkehr Oesterreiches ins Reich. " («Le jour de l'annexion de l'Autriche par le Reich") Le premier jugement sur Naven était beaucoup plus critique : voir son compte rendu dans Archiv für Anthropologie und Völkerforschung N.F. Bd. 24, 1938: 164-165. 
9. Au début des années 1960, l'historien des religions Angelo Brelich suggéra au cours d'une de nos conversations que De Martino avait essayé de saisir " une frange d'ultrahistoire» (una frangia di ultrastoria). Sur la trajectoire intellectuelle qui conduit De Martino à la rédaction du Monde magique. voir Carlo Ginzburg, "Momigliano e De Martino ", Rivista storica italiana 100, 1988: 400-413.

10. Pour une comparaison avec la Dialectique de la raison de Theodor $\mathrm{W}$. Adorno et Max Horkheimer. voir Ginzburg 1979 : 228-248. comme un reliquat du XIXe siècle (Shirokogoroff 1940). Ses descriptions ethnographiques minutieuses des pratiques chamaniques chez les Toungouses visaient à renverser, avec mépris, les présupposés traditionnels de la discipline. Le "folklore", ne cessait-il de répéter, était un savoir scientifique et la science, c'était le «folklore européen». II est aujourd'hui facile de passer à côté du caractère subversif de la remarque que voici:

Dans la mesure où le chamane se sert de son intuition pour «trouver les causes", il ne diffère en rien de n'importe quel autre individu qui adopte la méthode consistant à briser les usages culturels ordinaires comme moyen pour aller du connu à l'inconnu. (Shirokogoroff 1999: 360)

Plus haut, Shirokogoroff avait écrit: «Les Toungouses disposent de nombreux "faits" corroborant leur hypothèse" sur l'existence des ojan, c'est-à-dire des esprits (ibid. : 141).

À la lumière de cette référence aux «faits» entre guillemets, il est tentant de comparer The Psychomental Complex à un autre ouvrage, également publié en 1935 à Bâle: Genèse et développement d'un fait scientifique [Entstehung und Entwicklung einer wissenschaftlichen Tatsache] de Ludwik Fleck, qui a acquis une célébrité posthume à travers la Structure des révolutions scientifiques de Thomas Kuhn. II m'est, pour le moment, impossible d'affirmer que le relativisme radical de Shirokogoroff et celui de Fleck sont des développements parallèles, à partir d'une source commune. Mais, quoi qu'il en soit, les "faits" que Shirokogoroff, pour satisfaire aux exigences de son relativisme radical, a enregistrés de façon détaillée, De Martino les a intégrés à sa propre perspective. Je précise ici que je ne partage pas (et n'ai jamais partagé) l'intérêt de De Martino pour la parapsychologie, pas plus que ses efforts pour démontrer la réalité des pouvoirs magiques. Mais je me suis (et suis encore) vivement intéressé à l'argumentation théorique audacieuse du Monde magique, à savoir que la réalité et notre présence à elle sont le résultat d'un long processus historique dans lequel la magie a joué un rôle fondamental.

Comme le montrent les données ethnographiques collectées sur le terrain auprès de cultures différentes, souligne De Martino, les êtres humains, quand ils sont confrontés à des situations hautement critiques, sont menacés par une "perte de présence»: ils risquent d'être submergés dans le monde. Dans ces situations de crise, le chamane agit comme un héros culturel pour rétablir leur présence au monde. La performance chamanique est le reliquat d'un passé non remémoré. Le Monde magique constitue à cet égard une tentative pour recouvrer ce passé, atteindre cette strate pré-historique qui rendrait possible l'histoire humaine ${ }^{9}$. La démonstration démartinienne, tout en étant purement spéculative, est extrêmement stimulante ${ }^{\mathbf{1 0}}$

II est aujourd'hui facile de déceler dans Le Monde magique une forte dimension d'identification personnelle pleinement consciente avec le chamane et son univers. De Martino était séduit par l'approche de Shirokogoroff non seulement pour ses qualités intellectuelles intrinsèques, mais également parce que ce dernier rejetait les interprétations de ses prédécesseurs 
en termes de pathologie, «l'hystérie arctique ${ }^{\mathbf{1 1}}$ ». En transformant la science européenne en folklore européen et le chamane en héros culturel, Shirokogoroff ouvrait la voie à De Martino.

3. Dès son premier livre, Naturalisme et historicisme en ethnologie, De Martino s'était identifié à l'«historicisme » de Benedetto Croce, une perspective selon lui capable de conquérir de nouveaux territoires intellectuels, à commencer par l'ethnologie. Mais cette allégeance déclarée à Croce masque une trajectoire plus complexe et tortueuse, qui inclut des éléments aussi divers que la parapsychologie, l'existentialisme et, plus tard, les cahiers d'Antonio Gramsci (Ginzburg 2013). On a fait observer, par exemple, qu'une remarque critique à l'égard de la philosophie de Martin Heidegger, dans une note du Monde magique, semblait trahir une attitude ambivalente. Mais j'avancerai ici une hypothèse préalable: la première rencontre de De Martino avec Heidegger a pu avoir lieu à travers Mühlmann. En effet, celui-ci soulignait, dans Methodik der Völkerkunde, que la relation de l'individu à son «monde», en tant que problème existentiel, était au cœur de la philosophie heideggérienne ${ }^{\mathbf{1 2}}$. Et il citait partiellement le passage d'Etre et Temps auquel il se référait en particulier. Le voici:

Croire à la réalité du «monde extérieur », à tort ou à raison; prouver cette réalité, de façon satisfaisante ou non; la présupposer, explicitement ou non - autant de tentatives qui, n'ayant pas sur leurs propres bases une complète clarté, présupposent un sujet d'abord sans monde ou manquant d'assurance quant à son monde, un sujet qui doit au fond commencer par s'assurer d'un monde. (Heidegger 1986: 256, § 43a)

De Martino a pu considérer la citation elle-même - « un sujet d'abord sans monde ou manquant d'assurance quant à son monde, un sujet qui doit au fond commencer par s'assurer d'un monde" - comme une formule condensée du projet de recherche qu'il était alors en train d'élaborer. Quelques années plus tard, dans la note du Monde magique mentionnée plus haut, il rappelait sa dette intellectuelle envers Heidegger, tout en accentuant sa prise de distance: «L'existentialisme a identifié [...] un problème irrésolu du rationalisme moderne » [à savoir, la philosophie de Croce], «l'individu en tant que donné ", en tant qu'entité tenue pour assurée. Mais, poursuivait De Martino, Heidegger a abusivement étendu sa critique (qui était donc une juste critique) au point de rejeter toutes les formes de rationalisme. Au lieu de s'attaquer au problème théorique soulevé par «l'individu en tant que donné », il a promu «à la dignité d'une pensée l'expérience de la crise, aussi passionnément fût-elle vécue». À travers I'histoire de la magie, objectait De Martino, «la raison historique reprend ses droits» (De Martino 2003: 206-207, note 1).

Voilà comment De Martino percevait son propre livre. Mais il y avait un non-dit derrière ces mots: Shirokogoroff et Heidegger, ethnographes et missionnaires, lui avaient permis d'élaborer une expérience personnelle et intime, en tant que telle impossible à formuler. Cette trajectoire intellectuelle avait ouvert la possibilité d'aborder une question restée sans réponse dans la philosophie de Croce. La présence de l'individu dans le monde,
11. Pour une perspective opposée, voir Wilhelm Emil Mühlmann, «Exkurs: 'Arktische hysterie' und Schamanismus ", Chiliasmus und Nativismus, Berlin, Dietrich Reiner Verlag, 1961: 210-220. L'édition allemande est complétée par des essais de plusieurs auteurs, la traduction française est une version abrégée: Messianismes révolutionnaires du tiers monde, trad. Jean Baudrillard, Paris, Gallimard, 1968 Mühlmann s'appuie également sur le travail de Shirokogoroff.

\section{double page suivante}

fig. 3

Fernand Léger, La fin du monde filmée par l'Ange Notre-Dame de Blaise Cendrars, 1919, édition de La Sirène. Bon à tirer sur la première page, rehauts à l'aquarelle, $32 \times 50 \mathrm{~cm}$. Biot, musée national Fernand Léger, don galerie Jeanne Bucher (Paris) en 1995.

Photo (C) RMN-Grand Palais (musée Fernand Léger) / Gérard Blot. ADAGP, Paris, 2016.
12. Wilhelm Emil

Mühlmann, Methodik der Völkerkunde, op. cit. : 99; "La querelle qui s'enflamme autour de la philosophie existentielle de M. Heidegger montre l'acuité de la question du rapport de l'homme à son "monde", vécu comme un problème existentiel, qui occupe les esprits aujourd'hui. Ce philosophe considère l'attachement inexorable de l'homme à son monde comme une catégorie fondamentale de la vie dans son historicité, ce qui est aussi notre point de vue, mais plus encore comme une catégorie de l'être, passant ainsi de l'épistémologie à l'ontologie. II réfute par conséquent toute tentative visant à apporter la preuve d'une réalité du monde extérieur, étant donné que de telles tentatives présupposent "un sujet dépourvu de monde pour ne pas dire un sujet doutant de son monde, un sujet qui, au fond, au préalable doit s'assurer de son monde". C'est en ce sens que Heidegger conçoit l'"être-au-monde" comme totalité structurelle. " (Sein und Zeit : 203-208, vgl. 57 f.). Traduction de l'allemand par Rachelle Viennot Hüwel. 


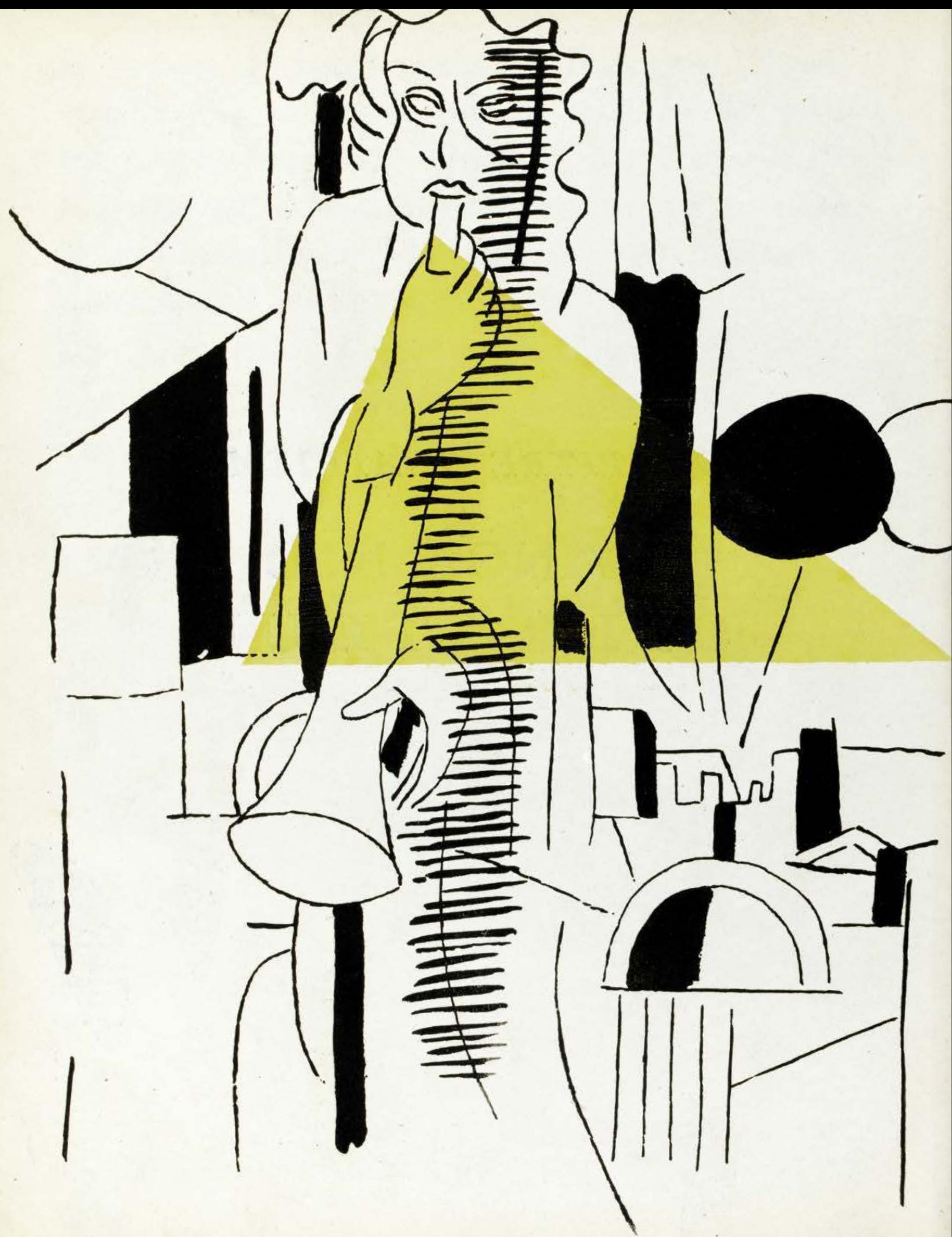




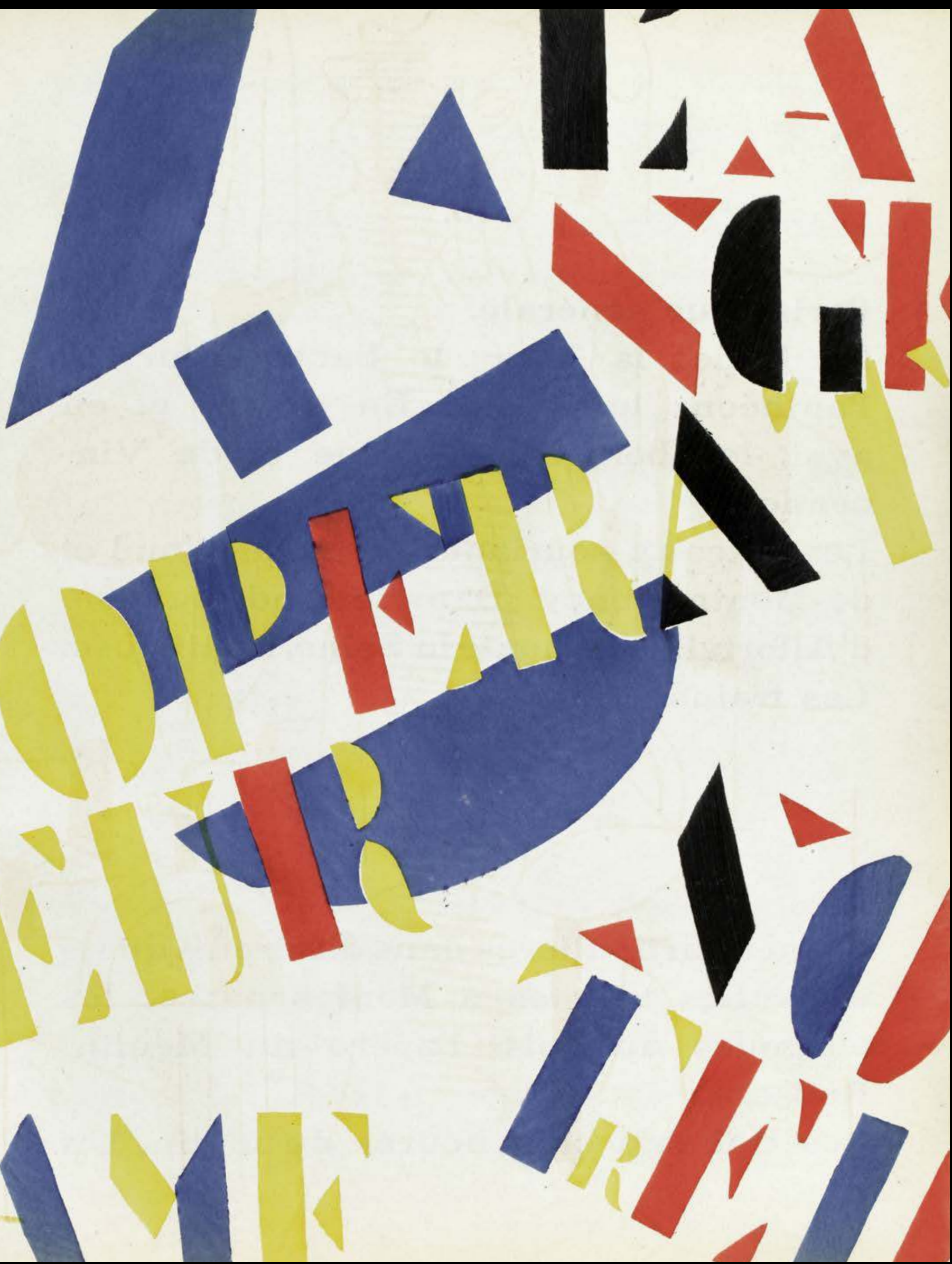


13. Repris sous le titre "Mythe et existence" in Enzo Paci, // nulla

e il problema dell'uomo [Le néant et le problème de l'homme], Turin, Taylor, 1950: 109-133, voir en particulier p. 123 sqq., ainsi que p. 130-131: «De Martino ne pense-t-il pas, malgré sa référence peu bienveillante à Heidegger [...], devoir quelque chose à l'existentialisme? »

14. "L'interlocuteur le plus important de De Martino dans les dernières années de sa vie", écrit Roberto Pàstina, l'éditeur de De Martino, Scritti filosofici, Bologne, II Mulino, 2005: VIII, note 2 .
15. Benedetto Croce, compte rendu de Studi filosofici, II, 1941, in La Critica 40, 1942: 48-49; "Ancora intorno alla vitalità come momento dello spirito" ["Encore sur la vitalité comme moment de l'esprit »], Quaderni della Critica 5(15), 1949: 93-95; id., " Il concetto dell'utile o del vitale e le sue aporie " ["Le concept d'utile ou de vital et ses apories »], Quaderni della Critica 5(14), 1949: 126.

16. "Et ce ne fut certainement pas le moindre mérite de Croce que d'avoir intégré dans la vie de l'esprit, traditionnellement limitée à la triade du bien, du beau et du vrai, le monde de l'utile comme valeur autonome (de ce point de vue, je crois qu'il faut réintégrer le rapport entre Croce et Marx et développer à nouveau la polémique entre Croce et Paci » ainsi que «reprendre la méditation sur l'utile " (De Martino 1977 : 642; traduction de l'italien par Giordana Charuty, ainsi que les autres citations, voir notes 23 et 24).

\section{ci-contre}

fig. 4

Fernand Léger, La fin du monde filmée par l'Ange Notre-Dame de Blaise Cendrars, 1919, édition de La Sirène. Bon à tirer sur la première page, rehauts à l'aquarelle, 32 × 25 cm. Biot, musée national Fernand Léger, Don Galerie Jeanne Bucher (Paris) en 1995. Photo (C) RMN-Grand Palais (musée Fernand Léger) / Gérard Blot. ADAGP, Paris, 2016. la présence comme prérequis de la vie spirituelle est, selon De Martino, un fait historique, une conquête historique.

4. On a déjà beaucoup écrit sur la dette intellectuelle de De Martino à l'égard de Heidegger (Cherchi 1987). Le premier à l'avoir mise en évidence est le philosophe italien Enzo Paci dans un compte rendu très éclairant du Monde magique ${ }^{\mathbf{1 3}}$. Dans les dernières années de la vie de De Martino, dominées par le projet inachevé de La Fin du monde, Paci occupe à nouveau une place particulière ${ }^{\mathbf{1 4}}$. Ici encore, De Martino suivait une piste qui avait été ouverte par Croce. Âgé alors d'une trentaine d'années (il était né en 1911, soit trois ans après De Martino), Paci était entré en dialogue avec Croce au début des années 1940 (Sini 2015: 28 sqq.). Rendant compte de son essai «La signification historique de l'existentialisme», Croce s'était montré étonnement réceptif aux objections soulevées par un jeune homme de presque cinquante ans son cadet. À celui qui avait, jadis, distingué quatre formes irréductibles de vie spirituelle - économique, pratique, esthétique et logique -, Paci faisait remarquer, en 1941, que la forme «économique» équivalait, de fait, à l'existence. Finalement, Croce accepta ce point de vue en faisant de la vitalité un synonyme de l'économique, c'est-à-dire une catégorie qui préexisterait à toutes les autres, et en laissant la voie ouverte à des spécifications ultérieures ${ }^{15}$. Entre temps, dans un article pensé comme une sorte de testament spirituel, il reconnaissait l'accent porté sur l'économique comme étant sa contribution la plus décisive à la pensée philosophique, en lien avec ses premières réflexions sur le marxisme (Croce 1945).

Dans une note liée au projet de La Fin du monde, De Martino insistait sur l'importance du «testament » de Croce comme de ses échanges avec Paci ${ }^{16}$. Pourtant, quelques années auparavant, dans le premier chapitre de Mort et lamentation rituelle dans le monde antique (1958), il formulait des critiques explicites à l'égard de la dernière philosophie crocienne. En mettant l'accent sur le rôle primordial de la «vitalité » dans la vie spirituelle, Croce avait attribué un rôle formel à la vitalité en tant que matière, donc brouillant la distinction entre les deux (De Martino 2000 [1958]: 17-18; Berardini 2012). La forme qui domine la vie spirituelle, comme il le soutenait auparavant dans L'Histoire comme pensée et comme action, est l'éthique, rappelait De Martino qui ne se départit plus jamais de la notion d'«éthos du dépassement ", à savoir une « impulsion éthique à aller au-delà de toute situation donnée» (Clara Gallini dans son introduction à Mort et lamentation rituelle..., De Martino 2000 [1958]: XXI). Implicitement, De Martino transformait (et, selon moi, affaiblit) la thèse centrale du Monde magique en affirmant que la présence avait une dimension éthique fondamentale (ibid. : 14 sqq.).

5. Une série de fragments également rédigés en 1958, ou juste après, montre que le sous-texte des annotations démartiniennes porte, à nouveau, sur Heidegger. Ces écrits, édités à titre posthume par Marcello Massenzio sous le titre Les Fondements pour une théorie du sacré, faisaient référence à la section d'Être et Temps dont MühImann avait extrait le passage cité plus haut (De Martino 1995: 99-138 et l'introduction de Massenzio: 22-26). Le risque d'être perdu dans le monde, le risque de perte d'authenticité soulignés par Heidegger sont bien réels, écrivait De Martino. Puis il ajoutait un commentaire qui accentuait le caractère personnel de 


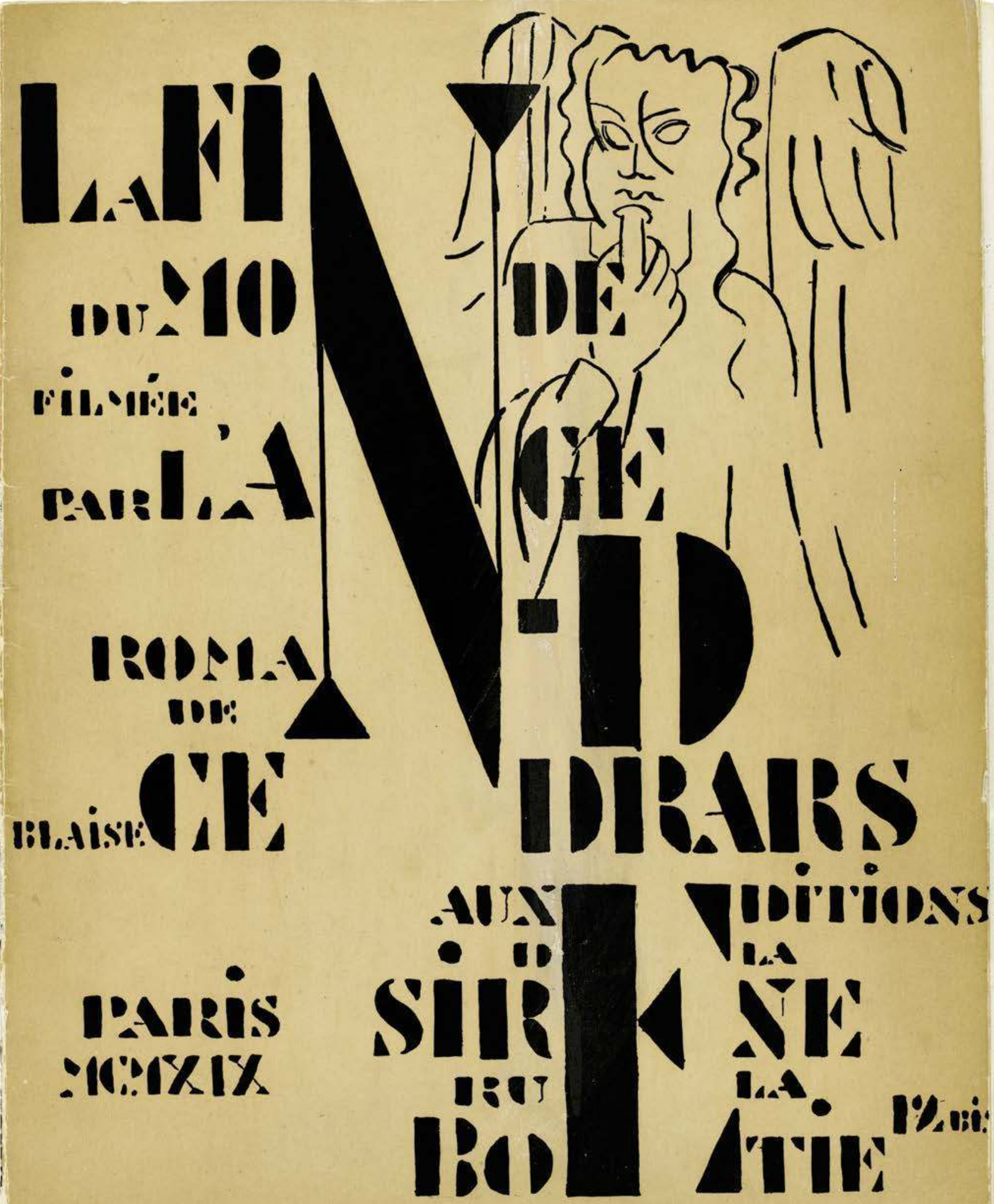


17. " ll y eut un moment crucial dans la conception de L'Éclipse quand, en février 1961, Antonioni filma une vraie éclipse de soleil à Florence ", David Saul Rosenfeld, «Endnotes", Michelangelo Antonioni's L'Eclisse: A Broken Piece of Wood, a Matchbook, a Woman, a Man, 2007 [en ligne], disponible sur: www.davidsaulrosenfeld com/endnotes.html.

18. L'Éclipse, dernière séquence: 9 minutes, 4 secondes. ses remarques: “Outre le caractère totalement inauthentique de l'épilepsie, il existe bien d'autres régimes d'inauthenticité. » (Ibid. : 121)

La philosophie de Heidegger, poursuivait De Martino en postulant que l'existence émerge du néant et court à la mort, «prend le risque pour la tâche, le danger pour le but, l'abysse pour l'alpiniste... » (ibid.). II fallait chercher la réponse dans l'éthos du dépassement, l'impulsion éthique à transcender la crise. L'avant-dernière philosophie de Croce fournissait ainsi une alternative à Heidegger. À ce point, le cadre conceptuel du projet de La Fin du monde était fixé; manquait encore l'étincelle de départ.

II.

1. La masse de dossiers publiée par Clara Gallini sous le titre La Fin du monde aurait dû inclure, a-t-on pensé, les notes rédigées par De Martino au début des années 1960 et publiées par Roberto Pàstina en 2005 sous le titre Écrits philosophiques. On trouve au début d'un premier ensemble de réflexions désigné par De Martino comme "cahier de Torre a Mare» une référence à l'article de Paci sur les rapports entre histoire et apocalypse chez Kierkegaard paru en 1954 (Paci 1954). La dernière référence bibliographique du cahier est La Pensée sauvage de Claude Lévi-Strauss, publiée en 1962, l'année qu'il faut considérer comme terminus ante quem non. Autour de cette date, quelque chose a dû déclencher la série de réflexions de De Martino sur l'apocalypse et les thèmes qui lui sont liés. Mon raisonnement conjectural ne se fondera pas sur un texte, mais sur un film: L'Éclipse de Michelangelo Antonioni, avec Monica Vitti et Alain Delon, qui sortit sur les écrans italiens le 12 avril 1962. En février 1961, Antonioni avait filmé une vraie éclipse de soleil à Florence ${ }^{\mathbf{1 7}}$. (Je me souviens avoir été témoin moi-même de ce spectacle inoubliable alors que j'étais à Pise.) Dans le film, l'éclipse devient la métaphore d'une attitude contemporaine morbide: la disparition des émotions et des sentiments, ou l'incapacité à les traduire. Ce thème de l'incommunicabilité était déjà au centre des deux premiers volets de la trilogie d'Antonioni, L'Avventura (1960) et La Nuit (1961). L'Éclipse ne connut pas un succès commercial, mais il fut ardemment discuté dans les milieux intellectuels. II n'est pas absurde de penser que De Martino ait pu voir le film, surtout si l'on se souvient d'une rencontre improbable, dont la presse avait fait grand bruit: Paci invita Antonioni et Monica Vitti (l'actrice principale du film, qui était aussi la compagne du réalisateur) à participer à un débat avec les étudiants de l'université de Milan (Paci 1963 et 2011).

Je n'ai jusqu'à présent parlé que du contexte: regardons maintenant le film - plus précisément la fin du film - pour vérifier s'il a pu avoir un impact sur le projet de De Martino, s'il a pu être une source d'inspiration. Mais d'abord, une remarque préliminaire. II y a dans les films d'Antonioni (en particulier ceux en noir et blanc) un contraste entre un scénario prétentieux et une intrigue sans intérêt d'un côté, et, de l'autre, une bouleversante beauté visuelle. L'Éclipse ne fait pas exception. L'intrigue peut se résumer très simplement: Vittoria, le personnage interprété par Monica Vitti, et Piero, joué par Alain Delon, ont une liaison. Ils décident de se revoir: aucun des deux ne vient au rendez-vous. Alors la fin (quelle fin?) commence ${ }^{\mathbf{1 8}}$. 
2. Une série de gros plans, apparemment sans lien entre eux, font lentement naître le sentiment qu'il va se produire quelque chose d'inexplicable et d'indicible: en regardant les dernières scènes de L'Éclipse, on est assailli par la sensation concrète d'une fin, pas seulement la lumière du jour qui décline - comme lors d'une éclipse de soleil - mais quelque chose de plus menaçant, qui fait signe vers la fin du monde. Cette dimension apocalyptique se lit dans les regards angoissés des individus qui marchent dans la rue et, plus directement encore, dans les gros titres d'un magazine que tient un voyageur descendant d'un bus: «La course à l'armement nucléaire», “La paix est fragile ${ }^{\mathbf{1 9}}$ ", deux articles parus dans le même numéro de L'Espresso du 10 septembre 1961 (Benci 2008). Ce détail renvoie à un contexte particulier, que les premiers spectateurs du film pouvaient immédiatement identifier: la crise suivant l'invasion manquée de Cuba par les Américains en avril 1961. Quelques mois plus tard, en octobre, l'installation de missiles soviétiques sur l'île avait failli déclencher une guerre nucléaire. Mais cet élément contextuel n'est qu'un détail d'une séquence où alternent humains et insectes, immeubles et arbres, vent et eau, tels les fragments d'une réalité apparemment dépourvue de sens. II n'y a pas d'intrigue: seulement la lente émergence de la nuit, qui finit par tout engloutir.

3. Cette lecture de la séquence finale de L'Éclipse n'est pas particulièrement originale. «Sur un plan visuel et auditif, peut-on lire dans un blog, les objets paraissent remplacer les formes humaines sans vie. Antonioni utilise également une série de gros plans très rapprochés comme un scientifique se servirait d'un microscope pour se rapprocher de la réalité. Un très gros plan sur l'oreille d'un vieil homme semble figer le visage, transformé en matière inerte. À la différence des objets qui s'animent soudain, tout mouvement humain semble être devenu impossible ${ }^{20}$. "Comme l'a justement souligné José Moure, auteur de plusieurs ouvrages consacrés à Antonioni, nous sommes face à «une sorte d'apocalypse» (Moure 1997 et 2001). Le lien que j'aimerais établir entre De Martino et Antonioni (idéalement véhiculé par Paci) est plus inattendu. II est possible que les images saisissantes d'Antonioni aient déclenché le projet de La Fin du monde, qui a fini par devenir une sorte de récapitulation de l'entière trajectoire de De Martino. Dans les dossiers dont il a déjà été question plus haut, il est fait une fois référence à l'éclipse de soleil, de façon indirecte, à travers l'expression «évidence du soleil». Chaque civilisation, écrit De Martino, réagit de manière différente à l'expérience d'une discontinuité de sa présence: dès que «le soleil réapparaît dans le contexte d'une action intentionnelle», sa perception culturelle doit être ajustée à une nouvelle situation humaine ${ }^{\mathbf{2 1}}$. (Ce qui rappelle la note de De Martino, citée plus haut, sur les crises d'épilepsie de sa jeunesse: «[Après la crise], la présence émerge du naufrage... ») À un niveau plus général, le contexte dans lequel prend place sa réflexion est le même que celui de L'Éclipse: avec la course à l'armement nucléaire, la fin du monde cessait d'être un mythe religieux et devenait réellement possible $\mathbf{2 2}$. Dans un commentaire de «Pour un temps d'apocalypse » d'Emmanuel Mounier, De Martino oppose d'une part «l'optimisme tragique » de l'apocalypse chrétienne, de l'autre, le sentiment de l'absurde lié à l'apocalypse sécularisée, engendrée par la crise moderne 23 (De Martino 1977: 334-335). À l'effondrement du monde fait écho l'effondrement du langage, parlé ou visuel. Comme ne cessait de le répéter De Martino, Le Journal d'une schizophrène
19. David Saul Rosenfeld, op. cit.

20. Rémi Bassaler, "Analysis of M. Antonioni's L'Eclisse (1962)", Dr. B or: How I Learned to Stop Worrying and Love Cinema, 2010 [en ligne], disponible sur: https:// doctorbcinema.wordpress. com/.../analysis-of-mantonionis-leclisse-1962.

21. "Mais dès que nous atteignons les limites de ses emplois culturels (que chaque civilisation, chaque régime d'existence définit différemment), l'astre solaire redevient objet d'intentionnalité, son histoire culturelle doit être adaptée à la situation critique du moment par une initiative plus ou moins "originale". » (De Martino 1977: 649-650)

22. L'allusion à Docteur Folamour (1964) de Stanley Kubrick est dans De Martino 1977 : 693. II s'agit du texte d'une communication pour un colloque.

23. À propos d'Emmanue Mounier ("Pour un temps d'apocalypse ", CEuvres, III, Paris, Seuil, 1962 : 341 sqq.) : "Face à l'activisme de l'apocalyptique chrétienne, qui relève d'un tragique optimisme, l'apocalyptique de la crise propre à notre actuelle conjoncture culturelle a changé de signe. [...] Le langage, au sens large d'un rapport sonore ou visuel entre moi et le monde, tend à suivre cet effondrement: ou plutôt le langage s'effondre lui-même pour se réduire, à terme, au silence et à l'incommunicabilité, au lieu d'être un appel intersubjectif. La maladie des objets, le sentiment de l'absurde, la perte de sens du monde, la nausée, etc. " 


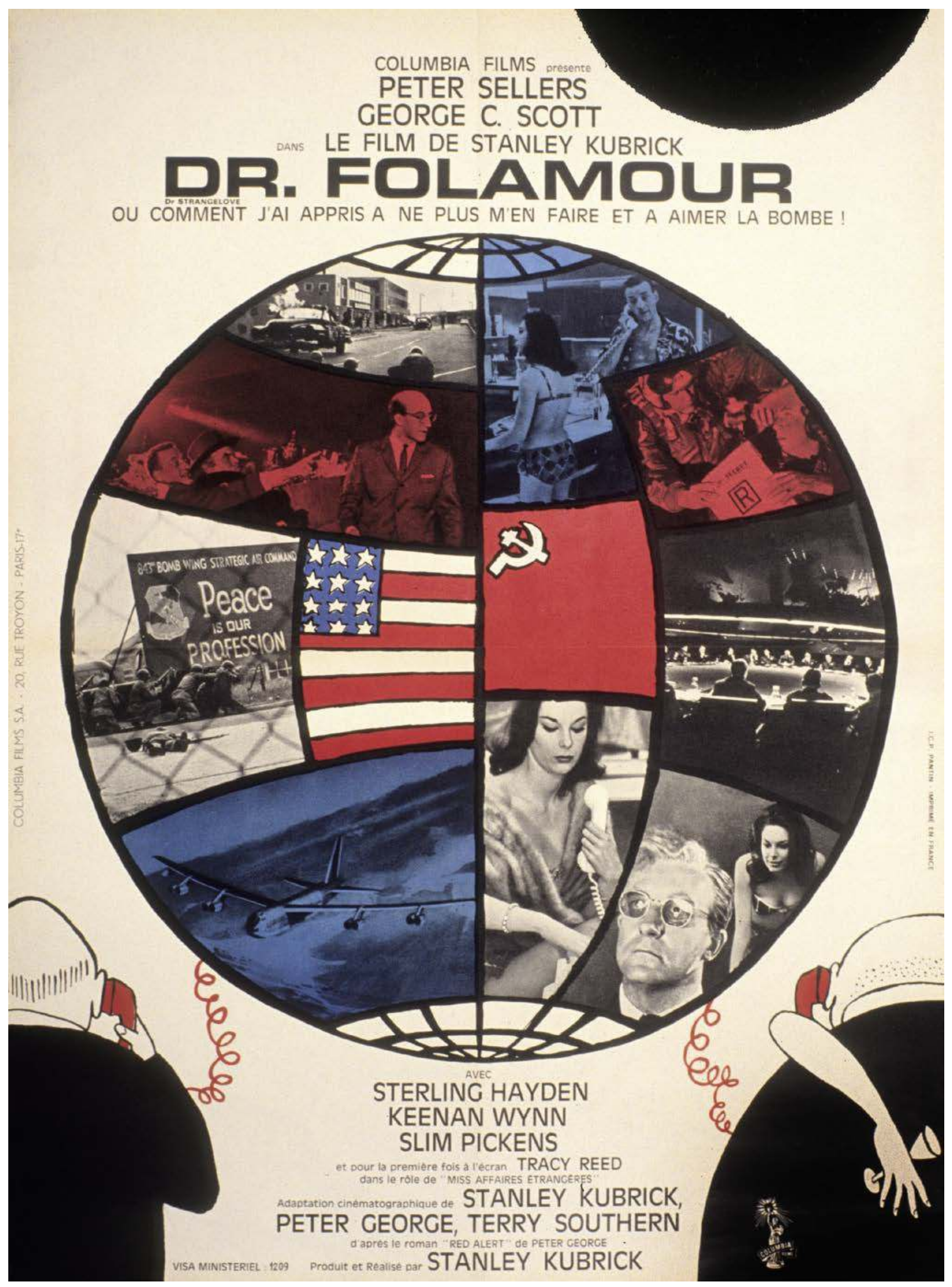


publié par Marguerite-Albert Sechehaye était révélateur car il fournissait l'exemple extrême d'une attitude beaucoup plus diffuse: la perte d'interaction émotionnelle avec la réalité environnante. Dans ce texte étonnant, Renée, la patiente de Sechehaye, décrivait un monde (son monde) dominé par une lumière froide et éblouissante. Le pays de la folie, le «pays de l'Éclairement », comme elle l'appelait, c'était «la perception de l'Irréalité». Tout dans ce pays était dans un état de tension, y compris elle-même: «C'est comme si un courant électrique d'une puissance extraordinaire passait sur toutes choses et tendait, tendait, jusqu'à ce que tout saute dans une explosion effroyable ${ }^{\mathbf{2 4}}$.» (Ibid.: 40-41) De Martino commente: "Nous sommes confrontés à une expérience terrifiante de l'univers sous tension, sur le point de s'anéantir dans une immense catastrophe. » (Ibid.: 41) Puis il ajoute ce qui ressemble à une description condensée de son projet de La Fin du monde: "Deux terreurs opposées dominent notre époque: celle de "perdre le monde" et celle d'"être perdu dans le monde". » (Ibid. : 475 sqq.)

Perte du monde et perte de la présence: pour De Martino, ce sont là les deux faces d'une même trajectoire émotionnelle et intellectuelle. L'exploration du lien entre elles est au cœur d'un essai intitulé «Apocalypses culturelles et apocalypses psychopathologiques », le seul article publié de son vivant où il anticipe le projet alors en cours (De Martino 1964: 131-132; et auparavant De Martino 1958: 54). On retrouve dans ce texte une citation de L'Ennui d'Alberto Moravia (1960) qui porte sur la question de l'«incommunicabilité", mot à la mode dans les milieux intellectuels de l'époque et le plus souvent associé aux films d'Antonioni25.

4. Au début des années 1960, dans un contexte historique très différent, De Martino se replongea dans des auteurs, morts (comme Pierre Janet) ou encore vivants (comme Mühlmann), qu'il avait lus vingt ans auparavant. En 1964, MühImann publie une nouvelle édition de Chiliasmus und Nativismus sur la «sociologie et la casuistique des mouvements révolutionnaires ". L'exemplaire conservé dans la bibliothèque de De Martino porte une dédicace de l'auteur et une date: «Rome, 24 avril 1965 ». De Martino mourait quelques jours plus tard ${ }^{26}$. Ses premières annotations soulignaient le caractère «très rudimentaire » (rozzezza estrema) de la perspective adoptée par l'auteur, qui assimilait les eschatologies à un «mélange» (Legierung) de traits normaux et pathologiques. On ne devait pas, soulignait De Martino, «expliquer le sain par le morbide»: «Il s'agit en revanche de considérer le lien organique (nesso organico) que les apocalypses culturelles manifestent entre risque psychopathologique et réintégration active, subordonnée aux valorisations communautaires de la vie humaine. » (De Martino 1977: 382)

Une fois de plus, De Martino mettait l'accent sur la plus ample portée du risque psychopathologique: une thématique qui, comme j'ai essayé de le montrer, constituait le véritable épicentre de sa trajectoire intellectuelle. La «réintégration active», inspirée par l'«éthos du dépassement» - le dépassement éthique de la situation existante -, est abondamment présente dans les notes sur La Fin du monde, comme elle l'était déjà dans Mort et lamentation rituelle. Je ne considère pas cette accentuation de l'éthique, plus prescriptive que descriptive, comme convaincante. «ll y a plus de choses
24. Citant MargueriteAlbert Sechehaye (Journal d'une schizophrène. Auto-observation d'une schizophrène pendant le traitement psychothérapique, Paris, Puf, 1950: 21) : “C'était cela, la folie, l'Éclairement c'était la perception de l'Irréalité. C'était se trouver d'une manière permanente dans l'irréalité la plus complète. Je l'appelais le "pays de l'Éclairement" à cause de la lumière éclatante, éblouissante et froide, astrale, et de l'état de tension extrême où se trouvaient toutes choses, y compris moimême. C'est comme si un courant électrique d'une puissance extraordinaire passait sur toutes choses et tendait, tendait, jusqu'à ce que tout saute dans une explosion effroyable. »

\section{ci-contre}

fig. 5

Affiche du Docteur Folamour, Stanley Kubrick, 1964 (C) Photo Rue des Archives/RDA.
25. Alberto Moravia (cité in De Martino 1964: 120): "Mais faites que le verre se décompose et perde sa consistance de la façon que j'imagine, ou bien qu'il se présente à mes yeux comme quelque chose d'étranger, avec lequel je n'ai aucun rapport, en un mot s'il m'apparaît un objet absurde, alors de cette absurdité jaillira l'ennui, lequel en fin de compte (c'est le moment de le dire) est le fait de l'incommunicabilité et de l'incapacité d'en sortir " (Alberto Moravia, L'Ennui, trad. Claude Poncet, Paris, Flammarion, 1986 [1961])

\section{Wilhelm Emil}

Mühlmann, Chiliasmus und Nativismus. Studien zue Psychologie, Soziologie und historischen Kasuistik der Umsturzbewegungen, Berlin, Dietrich Reimer, 1964. L'exemplaire conservé dans la bibliothèque de De Martino et qui se trouve aujourd'hui à la médiathèque de l'Académie de SainteCécile à Rome porte une dédicace manuscrite: "À mon cher collègue, le professeur De Martino, avec mon souvenir amical [Dem verehrten Kollegen Prof. De Martino zur freundlichen Erinnerung], Rome, 24 avril 1965 ». De Martino est mort le 6 mai 1965. 
sur la terre et dans le ciel, Horatio, qu'il n'en est rêvé dans votre philosophie»: on peut se demander si cette réplique de Hamlet que De Martino choisit comme épigraphe au Monde magique ne doit pas renvoyer, par-delà Croce, à De Martino lui-même. Sa contribution décisive à l'histoire et à l'anthropologie se situe dans sa profonde immersion analytique dans l'expérience de la crise, non dans son message positif - qui est là de toute façon. De Martino est une figure d'intellectuel tragique. En atténuer les traits contradictoires serait trahir son importance durable.

Université de Californie, Los Angeles - École normale supérieure de Pise ginzburg@history.ucla.edu

Traduction de l'anglais par Camille Joseph, revue par Giordana Charuty 


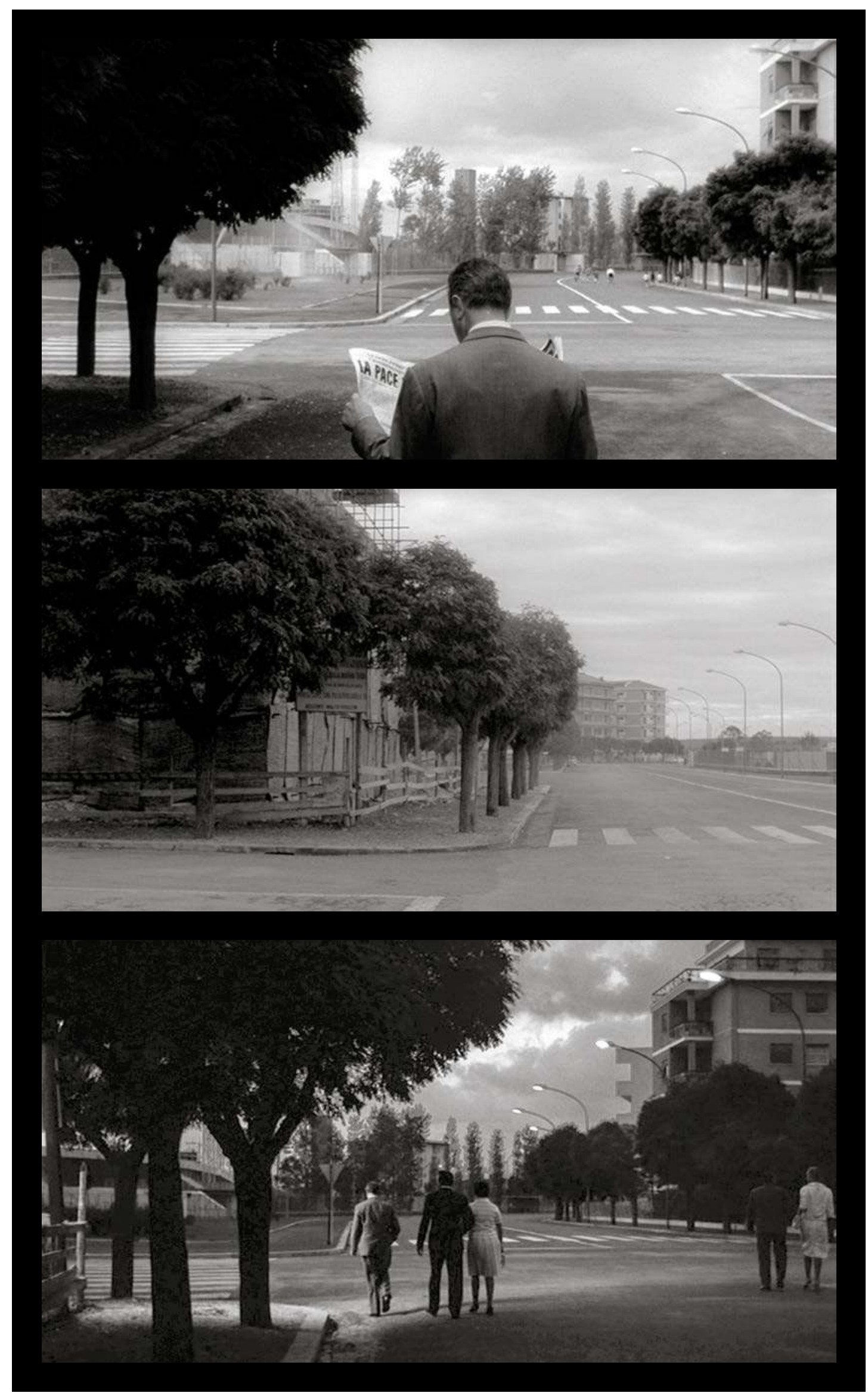




\section{Benci, Jacopo}

2008 «Michelangelo's Rome: Toward an iconology of the Eclisse" in Richard Wrigley (éd.), Cinematic Rome. Leicester, Troubador Publishing: 63-84

\section{Berardini, Sergio Fabio}

2012 L'Ethos della presenza. La ricerca filosofica di Ernesto De Martino [L'éthos du dépassement. La recherche philosophique d'Ernesto De Martino]. Université de Trente, thèse de doctorat.

\section{Charuty, Giordana}

2009 Ernesto De Martino. Les Vies antérieures d'un anthropologue. Marseille, Parenthèses-MMSH.

\section{Cherchi, Placido et Maria}

1987 Ernesto De Martino.

Naples, Liguori.

\section{Croce, Benedetto}

1945 «Vitalità ed esistenzialismo » ["Vitalité et existentialisme"], in "Note di storia della filosofia" XXV, Discorsi di varia filosofia, vol. II. Bari, Laterza: 291-292.

\section{De Martino, Ernesto}

1958 «Perdita della presenza e crisi del cordoglio" ["Perte de la présence et crise du deuil »], Nuovi Argomenti 30 : 49-92.

1964 "Apocalissi culturali e apocalissi psicopatologiche", Nuovi Argomenti 69-71: 105-141.

1977 La Fine del mondo. Contributo all'analisi delle apocalissi culturali, édition et introduction de Clara Gallini. Turin, Einaudi.

1995 Storia e metastoria. I fondamenti di una teoria del sacro, Marcello Massenzio (éd.). Lecce, Argo.

2000 [1958] Morte e pianto rituale. Dal lamento funebre antico al pianto di Maria. Turin, Bollato Boringhieri Editore.

2003 Le Monde magique. Paris, Les Empêcheurs de penser en rond.

\section{Ginzburg, Carlo}

1979 «La Fine del mondo d Ernesto De Martino ", Quaderni storici 40: 228-248.

2013 «De Martino, Gentile, Croce. Su una pagina di II mondo magico ", La Ricerca folklorica 67-68: 13-20.

\section{Heidegger, Martin}

1986 Etre et Temps, trad.

François Vezin. Paris, Gallimard.

\section{Moure, José}

1997 Vers une esthétique du vide au cinéma. Paris, L'Harmattan.

2001 Michelangelo Antonioni: cinéaste de l'événement.

Paris, L'Harmattan.

\section{MühImann, Wilhelm Emil}

1940 "Nachruf auf S.M.

Shirokogoroff (nebst briefllichen Erinnerungen) ", Archiv für

Anthropologie, Völkerforschung und kolonialen Kulturwandel N.F.Bd. 26: $55-64$.

\section{Paci, Enzo}

1954 «Storia ed apocalisse in Kierkegaard", Archivio di filosofia 17 («Apocalisse e insecuritas»): 141-162.

1963 «En torno a la dialéctica de la intersubjetividad ", Nuestro Cine 18: 4-7.

2011 "Enzo Paci and his students, a debate on Antonioni's L'Eclisse (1962) ", Annali d'italianistica 29: 33-58.

\section{Satta, Gino}

2005 «Le fonti ethnograpfiche de "Il mondo magico" ", in Clara Gallini (éd.), Ernesto De Martino e la formazione del suo pensiero. Note di metodo. Naples, Liguori : 57-77.

ia

\section{Shirokogoroff, Sergueï Mikhailovich}

1940 «Review of Lehrbuch der Völkerkunde by K. Th. Preuss, Stuttgart, 1937 ", in Archiv für Anthropologie N.F.B. 24(2): 158-161 (traduction anglaise de Wilhelm Emil Mühlmann).

1999 [1935] The Psychomental Complex of the Tungus. Berlin,

Reinhold Schletzer Verlag.

\section{Sini, Carlo}

2015 Enzo Paci. Milan, Feltrinelli.

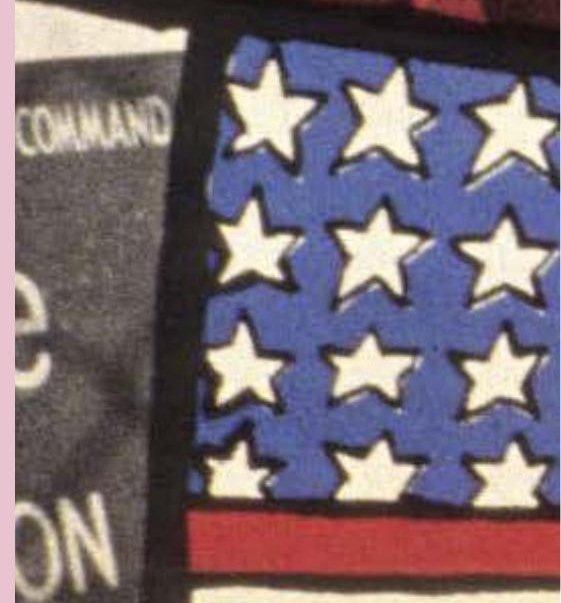




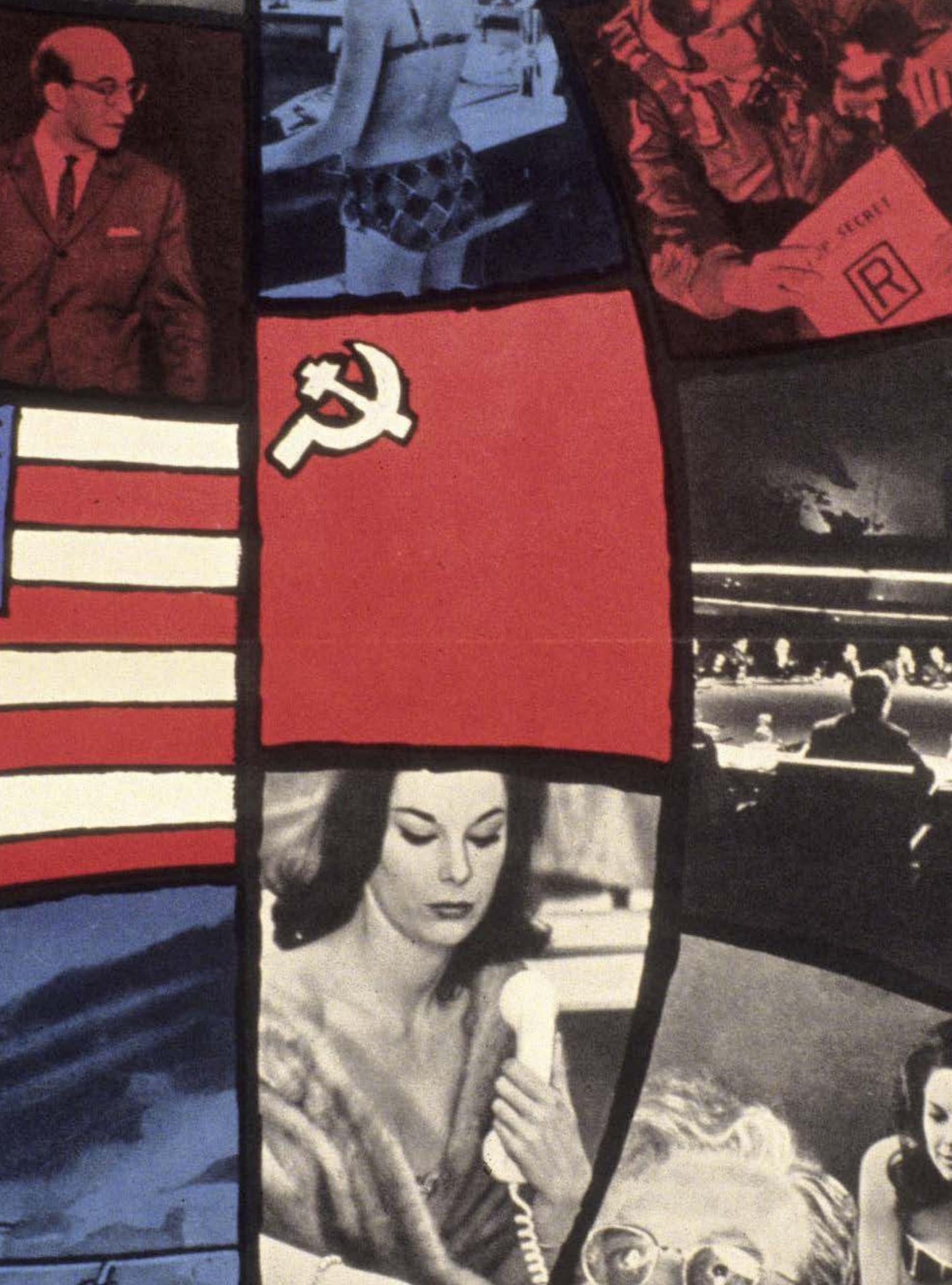

